\title{
Estimation of genetic parameters for temperament in Jeju crossbred horses
}

\author{
Nam Young Kim,a, Jun Kyu Son ${ }^{1, a}$, In Cheol Cho ${ }^{1}$, Sang Min Shin ${ }^{1}$, Seol Hwa Park ${ }^{1}$, Pil Nam Seong ${ }^{1}$, \\ Jae Hoon Woo ${ }^{1}$, Nam Geon Park', and Hee Bok Park ${ }^{1, *}$
}

\author{
* Corresponding Author: Hee Bok Park \\ Tel: +82-64-754-5719, Fax: +82-64-754-5713, \\ E-mail: heebokp@korea.kr \\ ${ }^{1}$ Subtropical Livestock Research Institute, National \\ Institute of Animal Science, RDA, Jeju 63242, Korea \\ a These authors contributed equally to this work.

\section{ORCID} \\ Nam Young Kim \\ https://orcid.org/0000-0002-2679-4983 \\ Jun Kyu Son \\ https://orcid.org/0000-0002-6266-3606 \\ In Cheol Cho \\ https://orcid.org/0000-0003-3459-1999 \\ Sang Min Shin \\ https://orcid.org/0000-0001-7924-1365 \\ Seol Hwa Park \\ https://orcid.org/0000-0002-7218-8212 \\ Pil Nam Seong \\ https://orcid.org/0000-0003-2915-1059 \\ Jae Hoon Woo \\ https://orcid.org/0000-0002-0332-142X \\ Nam Geon Park \\ https://orcid.org/0000-0001-6241-2850 \\ Hee Bok Park \\ https://orcid.org/0000-0002-9418-1898
}

Submitted Apr 5, 2017; Revised Jun 7, 2017; Accepted Mar 13, 2018
Objective: Temperament can be defined as a type of behavioral tendency that appears in a relatively stable manner in responses to various external stimuli over time. The aim of this study was to estimate genetic parameters for the records of temperament testing that are used to improve the temperament of Jeju crossbred (Jeju $\times$ Thoroughbred) horses.

Methods: This study was conducted using 205 horses (101 females and 104 males) produced between 2010 and 2015. The experimental animals were imprinted and tamed according to the Manual for Horse Taming and Evaluation for Therapeutic Riding Horses and evaluated according to the categories for temperament testing (gentleness, patience, aggressiveness, sensitivity, and friendliness) between 15 months and 18 months of age. Each category was scored on a five-point linear scale. Genetic parameters for the test categories were analyzed using a multi-trait mixed model with repeated records. The ASReml program was used to analyze the data.

Results: The heritability of gentleness, patience, aggressiveness, sensitivity and friendliness ranged from 0.08 to 0.53 . The standard errors of estimated heritability ranged from 0.13 to 0.17 . The test categories showed high genetic correlations with each other, ranging from 0.96 to 0.99 and high repeatability, ranging from 0.70 to 0.73 .

Conclusion: The results of this study showed that the test categories had moderate heritability and high genetic correlations, but additional studies may be necessary to use the results for the improvement programs of the temperament of Jeju crossbred horses.

Keywords: Temperament; Heritability; Repeatability; Genetic Correlation; Jeju Crossbred Horses

\section{INTRODUCTION}

A number of animal behavior studies have reported individual differences in behavioral characteristics, other than those associated with variables such as sex or age [1]. The behavioral responses expressed when an animal faces a specific situation are influenced by its temperament [1]. Temperament is regarded as a type of behavioral tendency that appears in a relatively stable manner across various situations and over time [2].

Horses have been genetically improved for various purposes, such as sports, leisure, and horseracing, and are categorized by morphological body confirmation or breed. The major traits for which horses have been improved include body size, racing speed, as well as strength, and some behavioral traits have also been enhanced. Temperament is regarded as an important factor to determine the usability of horses. For example, a survey of the members of the Pony Club in Australia, a youth organization that provides programs for horses and riding, reported that the temperament of horses (e.g., quietness, reliability, and stability) were more important traits for horses at the Pony Club than their body type, ridability or purchase 
price [3]. In addition, eleven Warmblood Horse Associations in Europe have established criteria for the temperament of horses and use them to evaluate horse breeds [4].

Multiple studies have been conducted to estimate genetic parameters for temperamental traits in livestock. Gauly et al [5] reported the possibility of selecting the temperament of cattle by estimating genetic parameters for temperamental traits in the German Angus and Simmental breeds. Lucena et al [6] also estimated heritability of temperamental traits in Nellora cattle. They reported moderate level of heritability for the temperamental trait [6]. In sheep, Zambra et al [7] demonstrated that the temperament of Uruguayan Merino and Corriedale sheep is moderately heritable. In cases of horses, there is a study estimated genetic parameters for the behavioral response of Thoroughbred horses at medical checkups for horseracing [8]. Another study estimated the heritability of the reactivity of Danish Warmblood horses in field tests [9]. The results showed that the heritability of temperament was estimated at a moderate level [10]. Thus, it is possible that systemic breeding programs could be implemented to improve the temperament of livestock including horses.

Jeju horse is a Korean native horse breed which lives in Jeju Island, Republic of Korea. Jeju horses have lived in Jeju Island more than 1,000 years and they possess several notable characteristics (e.g., height: 115 to $125 \mathrm{~cm}$; weight: 230 to $330 \mathrm{~kg}$; higher muscular endurance; robustness). The horses used in this study were crossbred between Jeju and Thoroughbred horses. This crossbred was established to use as a riding horse. The Jeju horse is described as a rather small to be used for riding, while Thoroughbred horse has a good body form for racing. Hence, we crossed the two horse breeds to generate a new synthetic breed which is appropriate for riding. We believe that the most important aspect for a riding horse is safety.
Thus, we intend to enhance temperamental traits directly associated with safety such as gentleness by collecting and analyzing temperamental trait data. This study was performed to estimate genetic parameters for the records of the temperament testing that are developed to improve the temperament of Jeju crossbred horses (Jeju $\times$ Thoroughbred).

\section{MATERIALS AND METHODS}

\section{Animals}

We established resource pedigrees by crossing Jeju and Thoroughbred horses. All the experimental animals were raised at the Subtropical Livestock Research Institute, the National Institute of Animal Science, Republic of Korea. For the $\mathrm{F}_{1}$ generation, we produced 163 animals ( 82 male, 81 female). The $\mathrm{F}_{1}$ progeny were then intercrossed to produce 37 (20 male, 17 female) $F_{2}$ progeny. In addition, $F_{1}$ male horses were backcrossed to Jeju mares, producing five horses (3 male, 2 female). A total of 205 horses from 6 sires and 88 dams were used for the estimation of genetic parameters. All the males were not castrated in this study. The experimental animals were produced between 2010 and 2015 (Table 1).

The horses used for analysis of the study were managed in individual horse stables with their dams for seven to ten days after delivery, and were imprinted and tamed during this period. The foals were then grazed with their mother horses until weaning. They were weaned three to four months after birth and raised in a horse barn as one group. The imprinting and taming was done according to the Manual for Horse Taming and Evaluation for Therapeutic Riding Horses [11]. This study was performed according to the animal care guide lines of the institutional Animal Care and Use Committee at the National Institute of Animal Science, Republic of Korea.

Table 1. Description of temperament characteristics

\begin{tabular}{ll}
\hline Classification & Description \\
\hline Gentleness & Tendency of staying in conformity while a horse is held by manager \\
& - Observe behavior when a horse is standing by itself \\
Patience & Capacity to endure with external stimuli or boredom \\
& - Observe how well a horse endures pain by gently pinching its neck and flank, or tapping on the body several times in a row; also, check if \\
the horse can endure about 30 minutes of boredom & Hostile status for a man or horse such as kicking or biting \\
Aggressiveness & - Observe if a horse behaves aggressively, such as biting or kicking, when approached by a person, another horse, or other animals \\
& - Observe if a horse scratches its forelegs, kicks the ground with its forelegs, shakes its head and body, or makes threatening sounds \\
& - Observe especially if a horse attacks from the back \\
& - Observe if a horse jumps excessively without a special reason and if it can be controlled \\
Sensitivity & Reaction to circumstance, sound and object etc. \\
& - Observe if a horse frequently looks around and reacts to the movement of a small object or faint sound \\
& - Observe a horse's reaction when it is approached by new objects on its body or close to its face \\
Friendliness & - Observe how a horse reacts when it hears a sudden loud noise or the sound of many people clapping \\
& - Observe the reaction when a horse is approached by a person acting violently and screaming
\end{tabular}




\section{Behavioral data collection}

The temperament of the experimental animals was evaluated when they were between 15 months and 18 months of age. The temperament evaluation was carried out annually, and three or more horse managers with at least one year of experience evaluated the categories for temperament testing from the Manual for Horse Taming and Evaluation for Therapeutic Riding Horses [11]. The horse managers assigned scores to each horse for the test categories of gentleness, patience and friendliness on a five-point linear scale for each category. On the five-point linear scale, one point indicated "very bad," and five points indicated "very good." As for aggressiveness and sensitivity, five points indicated the lowest tendency of aggressiveness and sensitivity. Therefore, we can also regard these individuals with five points in the aggressiveness and sensitivity tests as mild individuals (Table 1). The temperament of each horse (age: 15 to 18 months) was simultaneously assessed once by the 3 to 4 evaluators. When the horses were evaluated in an open space, each horse was held by each manager.

The record regarding the temperament of 205 horses produced between 2010 and 2015 was assessed by seven evaluators. Six hundred fifty nine records for gentleness, patience, aggressiveness and sensitivity, and five hundred thirty six records for friendliness, were used for the statistical analysis (Table 2). With regard to the five categories for temperamental traits investigated in this study, the mean and standard deviation of each category are shown in Table 3.

\section{Statistical analysis}

The ASReml program was used to estimate the genetic parameters and genetic correlations of each temperamental trait examined in this study (VSN international, Hemel Hempstead, UK) and a multi-trait mixed model was used for analysis as follows:

$$
\begin{aligned}
Y_{i j k l m}= & \mu+\text { sex }_{i}+\text { year }_{j}+\text { evaluator }_{k}+\text { year } \times \text { evaluator }_{j k} \\
& +a_{l}+p e_{l}+e_{i j k l m}
\end{aligned}
$$

Where, $Y_{i j k l m}=$ observations of each temperament trait, $\mu$ $=$ the overall mean, sex $x_{i}=$ the fixed effect of the ith sex $(i=$ male, female), year ${ }_{j}=$ the fixed effect of the jth birth year $(j=$

Table 2. Number of animals by each of the factors

\begin{tabular}{cccccccc}
\hline Year & No. & Sex & No. & Generation & No. & Parent & No. \\
\hline 2010 & 40 & Female & 101 & F1 & 163 & Sire & 6 \\
2011 & 35 & Male & 104 & F2 & 37 & Dam & 88 \\
2012 & 39 & & & BC $^{1)}$ & 5 & & \\
2013 & 45 & & & & & & \\
2014 & 25 & & & & & & \\
2015 & 21 & & & & & & \\
Total & 205 & & 205 & & 205 & & 94 \\
\hline
\end{tabular}

1) $F_{1}$ male horses were backcrossed to Jeju mares.
Table 3. Basic statistics of five temperament characteristics in the Jeju crossbred

\begin{tabular}{lcccccc}
\hline Trait & No. & Mean & SD & Min & Max & CV (\%) \\
\hline Gentleness & 659 & 3.83 & 0.98 & 1.0 & 5.0 & 25.6 \\
Patience & 659 & 3.41 & 1.14 & 1.0 & 5.0 & 33.4 \\
Aggressiveness & 659 & 3.69 & 1.11 & 1.0 & 5.0 & 30.1 \\
Sensitivity & 659 & 3.09 & 1.13 & 1.0 & 5.0 & 36.6 \\
Friendliness & 536 & 3.64 & 1.08 & 1.0 & 5.0 & 29.7 \\
\hline
\end{tabular}

SD, standard deviation; $C V$, coefficient of variation.

$1,2, \ldots, 6)$, evaluator ${ }_{k}=$ the fixed effect of the kth evaluator $(\mathrm{k}=1,2, \ldots, 7)$, year $\times$ evaluator $_{j k}=$ the fixed effect of year-byevaluator interaction, $a_{l}=$ the random additive genetic effect of the lth animal $\sim \mathrm{N}\left(0, \mathbf{A} \sigma_{\mathrm{a}}^{2}\right)$, where $\mathbf{A}$ is the numerator relationship matrix among animals, $p e_{l}=$ the random effect of the permanent environment $\sim \mathrm{N}\left(0, \mathbf{I} \sigma_{\mathrm{pe}}^{2}\right)$, and $e_{i j k l m}=$ the random residual effect $\sim \mathrm{N}\left(0, \mathbf{I} \sigma_{e}^{2}\right)$. The results of significance test of each fixed effect are shown in Supplementary Table S2. The predicted values ( \pm standard error) of year $\times$ evaluator are shown in Supplementary Table S3.

Heritability and repeatability using the variances estimated as above were obtained as follows:

$$
h^{2}=\frac{\sigma_{a}^{2}}{\sigma_{a}^{2}+\sigma_{p e}^{2}+\sigma_{e}^{2}} \text { and } r=\frac{\sigma_{a}^{2}+\sigma_{p e}^{2}}{\sigma_{a}^{2}+\sigma_{p e}^{2}+\sigma_{e}^{2}}
$$

Where, $h^{2}=$ heritability, $r=$ repeatability, $\sigma_{a}^{2}=$ additive genetic variance, $\sigma_{p e}^{2}=$ permanent environmental variance, and $\sigma_{e}^{2}=$ residual variance.

In addition, genetic and phenotypic correlation were estimated by:

$$
r_{G}=\frac{\operatorname{cov}_{a(x, y)}}{\sqrt{\sigma_{a(x)}^{2} \sigma_{a(y)}^{2}}} \text { and } r_{P}=\frac{\operatorname{cov}_{p(x, y)}}{\sqrt{\sigma_{p(x)}^{2} \sigma_{p(y)}^{2}}}
$$

Where, $r_{G}$ and $r_{P}$ were the estimates of genetic and phenotypic correlations. $C O V_{a(x, y)}$ and $\mathrm{COV}_{p(x, y)}$ were the genetic and phenotypic covariance components between a pair of traits $\mathrm{x}$ and $\mathrm{y}$, respectively. $\sigma_{a}^{2}$ and $\sigma_{p}^{2}$ were genetic and phenotypic variance components, respectively. Restricted maximum likelihood procedure was used to estimate the (co)variance components. We used log-likelihood convergence as the convergence criteria. The significance of the variance components were evaluated by likelihood ratio tests (Table 4 ).

\section{RESULTS AND DISCUSSION}

\section{Basic statistics}

With regard to the five categories for temperamental traits investigated in this study, the mean and standard deviation of each category are shown in Table 3 . The mean score of the test categories ranged from 3.09 to 3.83 . Given that the tem- 
Table 4. Variance components, heritability and repeatability for five temperament characteristics in the Jeju crossbred

\begin{tabular}{lccccc}
\hline Trait & $\sigma_{a}^{2}$ & $\sigma_{e}^{2}$ & $\sigma_{p e}^{2}$ & $h^{2} \pm \mathrm{SE}$ & $r \pm \mathrm{SE}$ \\
\hline Gentleness & $0.30^{(0.09)}$ & 0.28 & $0.36^{(0.01)}$ & $0.32 \pm 0.17$ & $0.71 \pm 0.03$ \\
Patience & $0.48^{(0.01)}$ & 0.36 & $0.35^{(0.02)}$ & $0.40 \pm 0.16$ & $0.70 \pm 0.03$ \\
Aggressiveness & $0.45^{(0.06)}$ & 0.34 & $0.34^{(0.05)}$ & $0.40 \pm 0.17$ & $0.70 \pm 0.03$ \\
Sensitivity & $0.67^{(0.001)}$ & 0.35 & $0.25^{(0.07)}$ & $0.53 \pm 0.15$ & $0.73 \pm 0.03$ \\
Friendliness & $0.09^{(0.32)}$ & 0.31 & $0.68^{(0.001)}$ & $0.08 \pm 0.13$ & $0.71 \pm 0.03$ \\
\hline
\end{tabular}

$\sigma_{a}^{2}$, genetic variance; $\sigma_{e}^{2}$, residual variance; $\sigma_{p e}^{2}$, permanent environmental variance; $h^{2}$, heritability; $r$, repeatability; SE, standard error.

Superscript numbers in parentheses refer to the nominal p-value obtained from likelihood ratio test to evaluate the significance of the corresponding variance component.

peramental trait "sensitivity" marked the lowest mean score, this trait should be of prime consideration in an attempt to improve the temperament of this study group. A temperament evaluation conducted by Kim et al [12] also showed that among the five temperamental traits, sensitivity marked the lowest mean scores with 2.9 points for female horses and 3.3 points for male horses. The present study also verified a similar result compared with the lowest score shown in sensitivity. The coefficient of variation (CV) for the test categories ranged from $25.6 \%$ to $36.6 \%$, and sensitivity showed the highest value of $36.6 \%$. The actual data did not follow normal distributions. However, the residuals of the data, obtained after the model fitting, showed normal distributions (Supplementary Figure S1).

\section{Heritability and repeatability estimations}

Table 4 presents the variance components and heritabilities of the data regarding gentleness, patience, aggressiveness, sensitivity, and friendliness in this study. The range of heritability (and standard error) for gentleness, patience, aggressiveness, sensitivity, and friendliness were 0.08 to 0.53 ( 0.13 to 0.17 ). These results indicate moderate heritabilities in gentleness, patience, and aggressiveness, low heritability in friendliness, and high heritability in sensitivity. The estimated heritability may be used as basic data for the genetic evaluation of the temperament in Jeju crossbred horses.

Oki et al [8] reported that the heritability of the behavioral response of Thoroughbred racehorses at medical checkups for racing ranged from 0.23 to 0.28 , which was similar with consideration of SE compared to the heritability levels for gentleness, patience, and aggressiveness in the present study. The repeatability levels of gentleness, patience, aggressiveness, sensitivity, and friendliness were $0.68,0.66,0.63,0.66$, and 0.69 , respectively. These results indicate high inter-evaluator reliability of the evaluated records.

\section{Phenotypic and genetic correlations}

Table 5 presents the genetic and phenotypic correlations of the data regarding the five categories used for testing the temperament of horses. The phenotypic correlation between gentleness and friendliness was 0.67 , and the phenotypic correlations between gentleness and aggressiveness, and between gentleness and sensitivity, were relatively high at 0.81 and 0.78 , respectively. The phenotypic correlation between patience and sensitivity was at 0.79 , but the phenotypic correlations between patience and aggressiveness, and between patience and friendliness, were at 0.77 and 0.61 , respectively. The phenotypic correlations between aggressiveness and sensitivity, aggressiveness and friendliness, and sensitivity and friendliness were at $0.78,0.65$, and 0.65 , respectively. The genetic correlations among the test categories were high, ranging from 0.96 to 0.99 .

We used the five-point linear scale (i.e., 1, 2, 3, 4, 5) for each testing category in this study. One point corresponded to "very bad", and five points corresponded to "very good" for gentleness, patience, and friendliness. When we evaluated aggressiveness and sensitivity, five points indicated the lowest tendency of aggressiveness and sensitivity. Therefore, we can also regard these individuals with five points in the aggressiveness and sensitivity tests as mild (gentle) individuals. And this can be the reason why we could obtain positive correlation coefficients between gentleness and the two traits.

Kim et al [12] reported that a principal component analysis on the categories for temperament testing resulted in only one factor. This finding is concordant to the results of the present

Table 5. Genetic and phenotypic correlation coefficients ( \pm standard error) for five temperament characteristics in the Jeju crossbred")

\begin{tabular}{lccccc}
\hline Trait & Gentleness & Patience & Aggressiveness & Sensitivity & Friendliness \\
\hline Gentleness & - & $0.99 \pm 0.04$ & $0.99 \pm 0.05$ & $0.98 \pm 0.06$ & $0.98 \pm 0.01$ \\
Patience & $0.75 \pm 0.03$ & - & $0.99 \pm 0.02$ & $0.99 \pm 0.04$ & $0.96 \pm 0.17$ \\
Aggressiveness & $0.81 \pm 0.02$ & $0.77 \pm 0.02$ & - & $0.99 \pm 0.06$ & $0.99 \pm 0.06$ \\
Sensitivity & $0.78 \pm 0.02$ & $0.79 \pm 0.02$ & $0.78 \pm 0.03$ & - & $0.97 \pm 0.12$ \\
Friendliness & $0.67 \pm 0.04$ & $0.61 \pm 0.04$ & $0.65 \pm 0.04$ & $0.65 \pm 0.04$ & - \\
\hline
\end{tabular}

\footnotetext{
1) Genetic correlations are above and phenotypic correlations are below the diagonal.
} 
study in which genetic correlations among the test categories were very high. Therefore, the five test categories used in this study may explain only a single main component. In practice, we don't need to continue to use all the five test categories. For example, we may omit friendliness due to the lack of significance in the genetic variance component. Additionally, since the sample size used in this study was moderate, a larger study should be conducted to estimate more precise genetic parameters.

In conclusion, the results of this study showed moderate heritability of the categories for temperament testing and high genetic correlations among the categories. However, additional research may be required to use these results for genetic improvement programs for temperament traits, and additional categories for temperament testing should be developed and established to carry out genetic evaluation of the temperament of various breeds of horses.

\section{CONFLICT OF INTEREST}

We certify that there is no conflict of interest with any financial organization regarding the material discussed in the manuscript.

\section{ACKNOWLEDGMENTS}

This work was carried out with the support of the Cooperative Research Program for Agriculture Science \& Technology Development (project title: Study of genetic characteristics evaluation in domestic riding horses, Project No. PJ01022201) by the Rural Development Administration, Republic of Korea.

\section{REFERENCES}

1. Seaman S, Davidson H, Waran N. How reliable is temperament assessment in the domestic horse (Equus caballus)? Appl Anim Behav Sci 2002;78:175-91.
2. Goldsmith HH, Buss AH, Plomin R, et al. Roundtable: What is temperament? Four approaches. Child Dev 1987;58:50529.

3. Buckley P, Dunn T, More SJ. Owners' perceptions of the health and performance of Pony Club horses in Australia. Prev Vet Med 2004;63:121-33.

4. Koenen E, Aldridge L, Philipsson J. An overview of breeding objectives for warmblood sport horses. Livest Prod Sci 2004; 88:77-84.

5. Gauly M, Mathiak H, Hoffmann K, Kraus M, Erhardt G. Estimating genetic variability in temperamental traits in German Angus and Simmental cattle. Appl Anim Behav Sci 2001;74: 109-19.

6. Lucena C, Neves HHDR, Carvalheiro R, Oliveira JAD, Queiroz SAD. Genetic analysis of the temperament of Nellore cattle using linear and threshold models. Animal 2015;9:388-94.

7. Zambra N, Gimeno D, Blache D, Van Lier E. Temperament and its heritability in Corriedale and Merino lambs. Animal 2015;9:373-9.

8. Oki H, Kusunose R, Nakaoka H, et al. Estimation of heritability and genetic correlation for behavioural responses by Gibbs sampling in the Thoroughbred racehorse. J Anim Breed Genet 2007;124:185-91.

9. Rothmann J, Christensen OF, Søndergaard E, Ladewig J. A note on the heritability of reactivity assessed at field tests for Danish Warmblood Horses. J Equine Vet Sci 2014;34:341-3.

10. Le Neindre P, Trillat G, Sapa J, et al. Individual differences in docility in Limousin cattle. J Anim Sci 1995;73:2249-53.

11.Lee JE, Kim NY, Kang DH, Jung KY, Kim TH. The Manual for horse taming and evaluation for therapeutic riding horses. Jointly published by National Institute of Animal Science, Republic of Korea and Samsung Equestrian Team, Republic of Korea. 2010 (Language: Korean, ISBN: 978-89-480-09200 93520).

12. Kim NY, Park YS, Ko MS, et al. Assessment of equine temperament by a questionnaire survey. J Emb Trans 2013;28:223-7. 